TRABAJO ORIGINAL

\title{
ADHERENCIA AL TRATAMIENTO EN PACIENTES CON DIABETES MELLITUS TIPO 2 EN ARGENTINA DURANTE 2015
}

\author{
ADHERENCE TO TREATMENT IN PATIENTS WITH \\ TYPE 2 DIABETES MELLITUS IN ARGENTINA DURING 2015
}

\begin{abstract}
María Amelia Linari ${ }^{1}$ Claudio González², Gustavo Frechtel ${ }^{3}$, Omar Álvaro ${ }^{4}$, María Inés Argerich ${ }^{5}$, María del Carmen Babus ${ }^{6}$, María Florencia Badia ${ }^{7}$, Leticia Barrera ${ }^{8}$, Natalia Blanco ${ }^{9}$, Diego Botta ${ }^{10}$, María Marta Curet ${ }^{11}$, Raúl David ${ }^{5}$, Alejandra Dib ${ }^{12}$, Guillermo Dieuzeide ${ }^{13}$, Daniel Dionisi ${ }^{9}$, Mariela Echenique ${ }^{14}$, Susana Fuentes ${ }^{15}$, Sandra Geraci ${ }^{16}$, Natalia Laguarde ${ }^{12}$, Silvia Lapertosa ${ }^{17}$, Astrid Libman ${ }^{18}$, Elizabeth Méndez ${ }^{9}$, Julieta Méndez ${ }^{19}$, Luis Neira ${ }^{12}$, María Florencia Noguero ${ }^{17}$, Verónica Ojeda ${ }^{20}$, Nelson Papini ${ }^{21}$, Víctor Previtera ${ }^{5}$, Erika Vago ${ }^{22}$, Carla Wassner ${ }^{23}$, Débora Chann ${ }^{24}$
\end{abstract}

\section{RESUMEN}

Introducción: la interrupción en la adherencia a los tratamientos prescriptos genera obstáculos importantes los cuales impactan negativamente en los indicadores de salud.

Objetivos: evaluar la adherencia al tratamiento en pacientes adultos con diabetes mellitus tipo 2 (DM2) en Argentina, y establecer la asociación con el tipo de prestación de atención en salud y la ubicación geográfica.

Materiales y métodos: se incluyeron 1.520 individuos pertenecientes a ocho regiones diferentes de la República Argentina durante 2015. Se realizó un estudio transversal. Se aplicó un cuestionario validado autorreferido de opciones múltiples con respuestas policotómicas de puntuación simple. Se empleó una escala cuantitativa y un análisis multivariado de componentes principales.

Resultados: la edad media fue de 60,2 años y la antigüedad de DM2 referida correspondió a 10,1 años. La media de HbA1c fue de $7,85 \%$. La media de adherencia general para todas las características fue 4,32 (61,71\%). Los resultados de adherencia encontrados se asemejan a otros países en desarrollo a excepción del cuidado de los pies. El factor cuidado de los pies en nuestro país reporta escalas de mayor adherencia. Se detectaron resultados tal vez redundantes como la mayor adherencia al monitoreo en las terapias de uso con insulina, y menores para dieta, ejercicio y solo uso de antidiabéticos orales (ADO), así como también la mayor adherencia en medicación en las terapias con insulina e insulina más ADO.

Conclusiones: son alarmantes las bajas escalas referidas al grupo con solo dieta y ejercicio.

Palabras clave: diabetes mellitus tipo 2; adultos; adherencia al tratamiento; prestación de atención en salud.

Revista de la Sociedad Argentina de Diabetes 2019; Vol. 53 (97-108)

\section{ABSTRACT}

Introduction: the interruption in the adherence to the prescribed treatments generates important obstacles which negatively impact on health indicators.

Objectives: to evaluate adherence to treatment in adult patients with diabetes mellitus type 2 (DT2) in Argentina; associate with the type of health care provision and location.

Materials and methods: we included 1.520 individuals belonging to eight different geographical regions of the Argentine Republic during 2015. A cross-sectional study was conducted. A self-reported validated questionnaire of multiple options with simple scoring polycotomic responses was applied. A quantitative scale and a multivariate analysis of main components were applied.

Results: the average age was 60.2 years and the seniority of the referred DT2 corresponded to 10.1 years. The average $\mathrm{HbA} 1 \mathrm{c}$ was $7.85 \%$. The average general adherence for all characteristics was $4.32(61.71 \%)$. The adherence results found are similar to other developing countries except for the care of the feet. The care factor of the feet in our country report scales of greater adherence. Redundant results are observed, such as greater adherence to monitoring in insulin therapy and less for diet and exercise and only oral antidiabetic drugs (ADO), as well as greater medication adherence in insulin and insulin therapies more ADO.

Conclusions: the low scales referred to the group with only diet and exercise are alarming.

Key words: type 2 diabetes mellitus; adults; adherence to treatment; delivery of health care.

Revista de la Sociedad Argentina de Diabetes 2019; Vol. 53 (97-108) 
1 Coordinación de la Sección Endocrinología y Nutrición, Unión Obrera Metalúrgica (UOM), Vicente López, Provincia de Buenos Aires, Argentina

2 Docente titular, Departamento de Farmacología de la Universidad Nacional de Buenos Aires, Ciudad Autónoma de Buenos Aires, Argentina

3 Coordinador del Servicio de Nutrición, Hospital Sirio Libanés, Ciudad Autónoma de Buenos Aires, Argentina

4 Coordinador Médico del staff en Centro Integral de Diabetes, Neuquén, Argentina

5 Médica/o de Planta, Hospital Perrupato, San Martín, Mendoza, Argentina

6 Médica de Planta, Centro Integral de Diabetes, Endocrinología y Nutrición (CIDEN), Jujuy, Argentina

7 Médica de Planta, Hospital Provincial de Rosario, Santa Fe, Argentina

8 Médica de Planta, Centro Médico Carrodilla y Obra Social de Empleados Públicos (OSEP), Maipú, Mendoza, Argentina

9 Médica/o de Planta, Hospital de Agudos Ramón Madariaga, Misiones, Argentina

10 Médico de Planta, Hospital Bouquet, Neuquén, Argentina

11 Médica de Planta, Hospital Regional Dr. Ramón Carrillo, Santiago del Estero, Argentina

12 Médica/o del Servicio de Nutrición, Hospital Sirio Libanés, Ciudad Autónoma de Buenos Aires, Argentina

13 Jefe del Servicio del Hospital Nuestra Sra. del Carmen de Chacabuco, Provincia de Buenos Aires, Argentina
14 Médica del staff de Centro Integral de Diabetes, Neuquén

15 Médica de Planta, Hospital El Cruce, Provincia de Buenos Aires, Argentina

16 Médica del Servicio de Nutrición, Hospital Castex de San Martín, Provincia de Buenos Aires, Argentina

17 Médica de Planta, Hospital Central J.R. Vidal, Corrientes, Argentina

18 Sanatorio Americano, Rosario, Santa Fe, Argentina

19 Médica de Planta, Hospital Vélez Sarsfield, Ciudad Autónoma de Buenos Aires, Argentina

20 Jefa del Servicio del Hospital Nacional de Clínicas, Córdoba, Argentina

21 Referente de Diabetes en el Área Departamental Luján de Cuyo, Mendoza, Argentina

22 Médica especialista, Centro Privado en Regina, Río Negro, Argentina

23 Médica del Servicio de Nutrición, Hospital Udaondo y Privado, Carapachay, Provincia de Buenos Aires, Argentina

24 Docente en la Universidad Tecnológica (UTN), Facultad Regional Buenos Aires, Ciudad Autónoma de Buenos Aires, Argentina

Contacto de la autora: María Amelia Linari

E-mail: marimelina@yahoo.com

Correspondencia: Liniers 1752 (CP1602), Florida, Provincia

de Buenos Aires, Argentina

Fecha de trabajo recibido: 09/02/19

Fecha de trabajo aceptado: 06/05/19

Conflictos de interés: los autores declaran que no existe conflicto de interés.

\section{INTRODUCCIÓN}

La Organización Mundial de la Salud (OMS) refiere que la deficiente adherencia al tratamiento de las enfermedades crónicas es un problema mundial de alarmante magnitud y a su vez considera que una tasa satisfactoria de adherencia al tratamiento no debe ser menor al $80 \%$. Este problema crece a medida que se incrementa la carga de la enfermedad crónica en el mundo; en consecuencia los tratamientos a largo plazo resultan con pobres resultados de salud y mayores costos sanitarios, mientras que los individuos con pocos recursos económicos son los más afectados. La mejora en la adherencia terapéutica aumenta la seguridad de los pacientes. En los países en desarrollo el promedio de la adherencia a los tratamientos a largo plazo alcanza sólo el $60 \%$, siendo menor en los países en vías de desarrollo. La evidencia permite observar que en promedio sólo uno de cada tres pacientes sigue de manera correcta las indicaciones de su médico, con adherencias diferentes para cada componente, mayores para la toma de medicamentos, aún en casos de polifarmacia y menores para ejercicio, dieta y monitoreo glucémico ${ }^{1,2}$.

El término "incumplimiento" al tratamiento culpabiliza al paciente que falla al momento de seguir las instrucciones médicas; sugiere una actitud pasiva y refleja connotaciones negativas. Por su parte, el término "adherencia" refiere al grado en el cual el comportamiento del paciente coincide con las recomendaciones acordadas con el profesional sanitario; lo mencionado resalta tanto la participación del paciente como la responsabilidad del médico y facilita decisiones compartidas.

Diversos factores se relacionan con la falta de adherencia como la falta de adherencia no intencionada o la falta de adherencia intencionada ${ }^{3,4}$. Los métodos y trabajos utilizados para evaluar la adherencia incluyen: indicadores de estado de salud, calificaciones de los proveedores de salud y observaciones de comportamiento como la motivación o autoinformes sobre los pacientes, entre otros. Un método de medición debe seleccionarse sobre la base de la fiabilidad, la validez, la sensibilidad al análisis de los comportamientos y la independencia en la medición de la condición de salud del paciente. La distribución de las mediciones debe basarse en la estabilidad de los comportamientos de adhesión y la congruencia temporal con otras medidas de interés como, por ejemplo, los objetivos de control glucémico.

Sobre una revisión de Cochrane se observó, en 83 intervenciones evaluadas a largo plazo para mejorar la adherencia, que sólo 25 se asociaron a un resultado clínico de impacto positivo. Casi todas las estrategias eran complejas incluyendo combinación 
de varias intervenciones. Incluso las más eficaces no implicaron grandes mejoras ni en la adherencia, ni en los resultados clínicos ${ }^{5}$. Posteriormente The National Institute for Health and Care Excellence (NICE), en otra revisión exhaustiva, no encontró evidencias claras sobre su eficacia ${ }^{6}$ respecto de las estrategias de intervención en el tema.

Los métodos de evaluación indirecta sobre la adherencia al tratamiento pueden basarse en: las entrevistas personalizadas, los cuestionarios autorreferidos, el control de retiro de recetas o de dispensaciones de medicación, incluso a través de la evaluación de los resultados clínicos esperados (descenso de la glucemia, alcance del cumplimiento de objetivos de colesterol, tensión arterial, etc. $)^{7,8}$.

El uso del cuestionario permite -en forma de escala de evaluación técnica- cuantificar y universalizar la información de manera económica y accesible para reclutar un número mayor de participantes y así facilitar el análisis ${ }^{9}$. Una de las encuestas más utilizadas en diabetes es la Morisky Medication Adherence Scale (MMAS-8) ${ }^{10,11}$. Otra herramienta aplicada es la escala cuantitativa Summary of Diabetes Self-Care Activities (SDSCA) ${ }^{12}$ la cual permite evaluar el cumplimiento de las recomendaciones indicadas en los siete días previos a la encuesta con respecto a los factores del cuidado de la diabetes (dieta, ejercicio, automonitoreo de glucemia, cuidado de pies y tabaquismo). La escala cuantitativa de adherencia representa el número de días en que el paciente cumplió con la actividad propuesta en el enunciado. Un puntaje de siete ( $7=100 \%)$ corresponde a la adherencia ideal y un puntaje de cero (0)a su ausencia total, a excepción de la variable tabaco donde se reporta a la inversa pues la variable de análisis es la presencia de tabaquismo.

Como antecedente para Argentina, con respecto a la aplicación del cuestionario SDSCA, se realizó un estudio en 176 afiliados adultos de la Unión Obrera Metalúrgica (UOM) con diabetes mellitus tipo 2 (DM2) durante 2013. El estudio demostró, en relación con la aplicación del cuestionario SDSCA ${ }^{13}$ aunado a otro validado vinculado a gastos en salud, constituir una medida multidimensional factible de evaluar autogestión, adherencia y viabilidad para extrapolarse en diferentes subpoblaciones con diabetes. Las escalas o puntuaciones más bajas de adherencia para el estudio de adherencia realizado en UOM 2013 correspondieron a la actividad física, el monitoreo glucémico y la dieta. Al asociar las variables de adherencia con el cumplimiento en el control metabólico, según objetivos individualizados, los resultados fueron significativos para la adherencia al monitoreo glucémico y los objetivos de control metabólico individualizados. En relación con los factores analizados en el estudio (dieta, ejercicio, monitoreo glucémico, cuidado de los pies, medicación y tabaquismo) no se encontró asociación entre la adherencia a los factores mencionados y la antigüedad del diagnóstico de la enfermedad, pero para esta asociación el número de analizados podría ser un limitante.

Es clara una asociación positiva constante entre la dificultad en la adherencia al tratamiento y el aumento del riesgo de muerte o eventos cardiovasculares. Sin embargo la lectura de los estudios, al buscar asociaciones, aún demuestra debilidades. No integrar la falta de adherencia al análisis clínico de un fracaso terapéutico conduce a la realización de pruebas innecesarias o a intensificar tratamientos que pueden aumentar los riesgos de los pacientes. La interrupción en la adherencia a los tratamientos prescriptos aún es un obstáculo importante para lograr los objetivos en pacientes con DM2.

El propósito de este trabajo es analizar y establecer la dimensión del problema que representa la adherencia al tratamiento en adultos con DM2 en Argentina según prestación en salud y región geográfica. Lo mencionado facilitará medidas válidas y confiables de evaluación de situación y estado actual en adultos con DM2 dentro de un contexto de apoyo científico y social que permita identificar las herramientas que el paciente, su núcleo familiar y el equipo de salud consideran lo apoyan, de modo tal de mejorar su potencial de adherencia y respuesta al tratamiento de la enfermedad.

\section{OBJETIVOS \\ Objetivo principal}

- Estimar la adherencia al tratamiento en pacientes adultos con DM2 en Argentina; establecer asociación con el tipo de prestación en salud y la ubicación geográfica.

\section{Objetivos específicos}

- Evaluar diferencias en la adherencia al tratamiento en adultos con DM2 en Argentina reportada por autorreferencia según prestación en salud y región geográfica a través de factores relativos al cuidado de la diabetes como: alimentación, actividad física, monitoreo glucémico, cuidado de los pies, consumo de cigarrillos y medicación prescripta. 
- Establecer asociación entre los factores de cuidado de DM2 relativos a la adherencia al tratamiento con antecedentes personales y características clínicas como: edad, sexo, antigüedad de la enfermedad, nivel de educación y tipo de tratamiento prescripto.

\section{MATERIALES Y MÉTODOS Diseño}

Estudio de tipo transversal.

\section{Población}

Pacientes adultos con DM2 que se atendieron en diferentes centros de atención ubicados en la República Argentina.

\section{Muestra}

Perteneció a ocho regiones geográficas de la República Argentina. Las regiones aplicadas replican los capítulos de trabajo que divide y trabaja la Sociedad Argentina de Diabetes (SAD). Sobre cada región geográfica se propuso reclutar no menos de 195 pacientes con DM2. Por cada grupo de características a reclutar, según tipo de prestación, el objetivo fue tomar 65 pacientes por cada centro de atención público o sin prestación, 65 pacientes por cada centro de atención privado o con prestación en salud u obra social, y 65 pacientes jubilados. Se incluyeron adultos mayores de 65 años jubilados, adultos con prestación en salud (prepaga, mutual u obra social) y adultos sin obra social. El número total propuesto de reclutados fue de 1.520. Las encuestas las respondieron en la sala de espera o en su domicilio para luego entregarlas en los lugares de atención. No se incluyeron pacientes con disglucemia.

Se incluyeron 1.520 pacientes adultos con diagnóstico de DM2 con tres meses o más de antigüedad de la enfermedad, previo consentimiento informado y firmado. El porcentual de no respuesta para el valor objetivo total correspondió a un 2,56\%. Los intervinientes en el reclutamiento de pacientes que pertenecían a las regiones integraron las siguientes provincias (Anexo):

- Provincia de Buenos Aires: se reclutaron 261 pacientes en total y se incluyeron aquellos sin obra social y jubilados en el Hospital Castex de San Martín, pacientes con obra social en el Policonsultorio Privado en Carapachay, sin obra social y jubilados en el Hospital Nuestra Señora del Carmen de Chacabuco y pacientes con obra social en el Centro de Atención e Investigación Privado en Chacabuco.
- Ciudad Autónoma de Buenos Aires (CABA): se reclutaron 167 pacientes en total y se incluyeron sin obra social pertenecientes al Hospital Vélez Sarsfield, con obra social pertenecientes en la atención a prestación privada en una clínica privada ubicada en CABA y pacientes jubilados con cápita en el Hospital Sirio Libanés.

- Comahue: se reclutaron 224 pacientes en total y se incluyeron aquellos con obra social, sin obra social y jubilados pertenecientes a las provincias de Neuquén y Río Negro.

- Centro: Córdoba reclutó 67 pacientes en total con obra social, sin obra social y jubilados.

- Cuyo: reclutó 229 pacientes en total e incluyó residentes en la provincia de Mendoza con obra social, sin obra social y jubilados.

- Litoral: reclutó 188 pacientes en total y sumó residentes en la provincia de Santa Fe con obra social, sin obra social y jubilados.

- Noreste: reclutó 244 pacientes en total con residentes en las provincias de Misiones y Corrientes con obra social, sin obra social y jubilados.

- Noroeste: reclutó 140 pacientes en total e incluyó residentes en las provincias de Jujuy y Santiago del Estero con obra social, sin obra social y jubilados.

\section{Instrumento de recolección}

Se realizó una encuesta adaptada que constó de dos partes ${ }^{13}$ :

- Parte 1: cuestionario rediseñado con datos demográficos, educacionales, socioeconómicos y clínicos de los pacientes (14 ítems autorreferidos de opciones múltiples con respuestas policotómicas de puntuación simple) $)^{14}$.

- Parte 2: el Summary of Diabetes Self-Care Activities (SDSCA) interroga sobre la adherencia al tratamiento en los siete días previos con una encuesta autorreferenciada de 14 ítems relacionados con la alimentación, la actividad física, el monitoreo glucémico, el cuidado de los pies, el consumo de cigarrillos, la medicación y el equipo de salud. Operativamente se utilizó una escala cuantitativa de adherencia de 0 a 7 representando el número de días en que el paciente cumplió con la actividad propuesta en el enunciado (un puntaje de 7 correspondió a adherencia ideal y un puntaje de 0 a su ausencia total) ${ }^{12}$.

\section{Variables}

Fueron adaptadas del estudio de tesis de maestría e incluyeron: 
- Aspectos personales. Se comprendieron edad, sexo, presencia y tipo de prestación de salud (sin obra social, con obra social y jubilación), fecha de diagnóstico de $\mathrm{DM} 2^{13}$.

- Aspectos educacionales. Relacionados con el nivel académico: no lee ni escribe, primario incompleto, primario completo, secundario incompleto, secundario completo, terciario o universitario incompleto, terciario o universitario completo. Para medir asociaciones con otras variables se colapsaron en cinco categorías: no lee ni escribe, primario incompleto, primario completo (incluye primario completo y secundario incompleto), secundario completo (incluye secundario completo y terciario o universitario incompleto), superior (terciario y universitario completo).

- Aspectos clínicos.

- Comorbilidades (antecedentes autorreferidos del paciente que le refirió en alguna oportunidad al profesional de la salud): hipertensión o tensión arterial elevada, presencia de eventos cardiovasculares (infarto, accidente cerebrovascular), dislipemia (colesterol alterado), esteatosis severa o hepatitis crónica o insuficiencia hepática, trastornos en la coagulación o enfermedad crónica de los glóbulos rojos.

- Complicaciones crónicas (antecedentes autorreferidos del paciente que le refirió en alguna oportunidad al profesional de la salud): oculares, renales, neuropatías.

- Control metabólico: hemoglobina glicosilada ( $\mathrm{HbA} 1 \mathrm{c})$, promedios de glucemias de los últimos tres meses según medidor de cada paciente (se consideraron los promedios de glucemia para el control metabólico en aquellos pacientes que refirieron complicaciones crónicas renales avanzadas, esteatosis grave, hepatitis crónica o insuficiencia hepática y enfermedades hemáticas) y cumplimiento de los objetivos glucémicos. Se definieron si cumplían o no con los objetivos de control glucémico o metabólico en forma individualizada. Para esto se categorizaron tres grupos según antecedentes y características como lo establecen los estándares de la Asociación Americana de Diabetes (ADA) y la Asociación Europea para el Estudio de la Diabetes (AEED) ${ }^{15}$.

Grupo 1: en pacientes con DM2 de reciente diagnóstico o menos de 10 años de conocido, sin antecedentes de hipoglucemia frecuente y/o grave, sin complicaciones crónicas de la enfermedad micro o macrovasculares, $\mathrm{HbA} 1 \mathrm{c}$ de $<7 \%$ o promedios de glucemia $<150 \mathrm{mg} / \mathrm{dl}$.

Grupo 2: en pacientes con DM2 con más de 10 años de antigüedad, antecedentes de hipoglucemia frecuente y/o grave, presencia de complicaciones micro o macrovasculares, $\mathrm{HbA} 1 \mathrm{c}$ entre 7-7,5\% o promedios de glucemia entre 151-180 mg/dl.

Grupo 3: en pacientes con DM2 con historia de hipoglucemias severas (pérdida de conocimiento o necesidad de ayuda de un segundo para el tratamiento), hipoglucemias asintomáticas, expectativa de vida limitada, condiciones comórbidas extensivas, complicaciones avanzadas (retinopatía severa, insuficiencia renal crónica establecida), analfabeto (no escribe ni lee) que haya faltado en el último trimestre a su trabajo como consecuencia de una descompensación o complicación diabética, jubilación prematura a causa de la diabetes, HbA1c 7,5-8,0\% o promedios de glucemia entre 181-200 mg/dl.

- Variables de adherencia. Se tuvieron en cuenta las dimensiones o factores incluidos por la OMS $2004^{1}$ referente a lo socioeconómico, los relacionados con el equipo o el sistema de salud, la asistencia sanitaria, la enfermedad propiamente dicha (factores relacionados con el tratamiento y el paciente). Se seleccionaron seis factores vinculados con el cuidado de la DM2: dieta o alimentación recomendada, ejercicio o actividad física sugerida, automonitoreo o monitoreo glucémico, cuidado de los pies, medicación recomendada y tabaquismo (se consideró fumador a quien lo refiriera). Se definió:

- Adherencia. Operativamente se utilizó cuestionario validado SDSCA $^{12}$, el cual se da en una escala cuantitativa de adherencia del 0 a 7 representando el número de días en que el paciente cumplió con la actividad propuesta en el enunciado (un puntaje de 7 correspondió a adherencia ideal y un puntaje de 0 a su ausencia total, excepción de la variable tabaco donde se reporta a la inversa). Es decir, los siete puntos ponderados o convertidos en porcentajes resultaron de la siguiente manera: $0=$ ausencia total, $1=15$ y $<30 \%$, $2=30$ y $<45 \%, 3=45 y<60 \%, 4=60 y<75 \%, 5=75$ $y<90 \%, 6=90$ y $<100 \%, 7=100 \%$

- Tipo de tratamiento: dieta y ejercicio, medicación oral o antidiabéticos orales (ADO), insulinoterapia, medicación oral e insulinoterapia.

\section{Análisis estadístico y herramientas utilizadas hasta el momento}

Se diseñó una ficha para la recolección de los datos y luego una base de datos en Excel. La información se procesó con InfoStat y en R. Se describieron características y se utilizaron datos 
expresados como media, desviación estándar, mediana y porcentajes.

Operativamente para evaluar el grado de adherencia reportada se utilizó SDSCA. Se asociaron los factores de adherencias a otras variables como: sexo, edad, antigüedad de DM, nivel de educación y cumplimiento de objetivos metabólicos.

Se asociaron los factores de adherencias al tipo de tratamiento: dieta y ejercicio solamente, antidiabéticos orales, insulina y antidiabéticos orales, insulina solamente y se analizaron diferencias estadísticas, medias, medianas, cuartil inferior y superior. Se aplicó test Kruskal Wallis.

Se realizó un estudio multivariado por medio del análisis de los componentes principales entre adherencia a la alimentación, actividad física, monitoreo glucémico, cuidado de los pies y características como el sexo, la antigüedad de la enfermedad y tipo de tratamiento de los encuestados con DM2. Se utilizaron grupos definidos por sexo y tipo de tratamiento. Es decir que se observó en cada punto del gráfico no un individuo de la muestra, sino un grupo de individuos del mismo sexo y con la misma recomendación de tratamiento. El objetivo fue evaluar un patrón de conducta respecto de la adherencia en función del sexo y el tratamiento de los pacientes. Para la construcción de este conjunto de variables se empleó la matriz de correlaciones de las variables originales.

\section{Reparo ético}

Se presentó el protocolo base realizado y aplicado en 2013 al Comité de Docencia e Investigación correspondiente a la Clínica Crespo, perteneciente a la Unión Obrera Metalúrgica, seccional Norte de Vicente López ${ }^{13}$, el cual fue en ese momento aprobado. Se indicaron el proyecto de investigación, la encuesta a utilizar, la nota de información al paciente, el consentimiento informado a cada uno de los centros de reclutamiento para analizar viabilidad y la aplicación del diseño de estudio transversal. En los centros de reclutamiento que fuera necesario se aguardó la autorización de la Dirección Médica de la institución o de los Comités de Docencia e Investigación correspondientes.

\section{RESULTADOS}

\section{Características y adherencias generales}

Sobre un total de 1.520 pacientes adultos de los cuales 802 eran mujeres y 685 hombres (no contestaron para el sexo 33 pacientes), la edad media resultó de 60,2 años y la antigüedad de DM2 referida correspondió a 10,1 años. La media de HbA1c fue de 7,85\% y las medias glucémicas de 150,34 $\mathrm{mg} / \mathrm{dl}$ (Anexo). La media de adherencia general para todas las características fue 4,32 (61,71\%). Los mejores puntajes en orden descendente fueron para las características: fumadores (M 5,63; DS 2,11 ; IC 5,53; 5,74), cuidado de los pies (M 5,22; DS 2,06; IC 5,12; 5,32) y adherencia a la medicación prescripta (M4,54; DS1,99; IC4,44; 4,64). En escala ascendente los peores puntajes fueron para la actividad física, el monitoreo glucémico y la alimentación (Figura 1 y Tabla 1).

\section{Adherencia según prestación en salud y región geográfica}

Se analizaron la adherencia y las diferencias estadísticas entre grupos según prestación en salud: con obra social, sin obra social y jubilado por medio de la prueba de Kruskal Wallis (Tabla 2). Para el cuidado en la alimentación se encontró diferencia significativa entre el grupo con obra social (mayor adherencia) vs los jubilados y sin obra social. Respecto de la actividad física, el grupo sin obra social (mejor adherencia) denotó diferencias significativas respecto de los jubilados y los con obra social. El monitoreo glucémico presentó diferencias significativas con mejor adherencia para el grupo con obra social y jubilados vs aquellos sin obra social. Para la escala sobre mejor adherencia antitabaco correspondió significativamente al grupo jubilados. En relación con la adherencia a la medicación prescripta la diferencia fue significativamente mejor en escala para el grupo con obra social y los jubilados vs los sin obra social.

Al analizar por región geográfica todas las adherencias cuantificaron en mejor escala para la variable antitabaco y cuidado de los pies (Tabla 3). Respecto de la adherencia a la medicación, resultó tercera en orden para todas las regiones a excepción de la región Cuyo. La adherencia a la práctica de actividad física fue el valor en escala menor para todas las regiones; además se hallaron diferencias por región para la adherencia al monitoreo glucémico y la alimentación.

Al asociar los factores de adherencias al tipo de tratamiento se aplicó test de Kruskal Wallis y se analizaron diferencias estadísticas, medias, medianas, cuartil inferior y superior (Tablas 4 y 5 ). Se observó mayor adherencia al monitoreo en las terapias de uso con insulina, y menores para dieta 
y ejercicio y sólo uso de ADO, así como también mayor adherencia en medicación en las terapias con insulina e insulina más ADO.

\section{Análisis multivariado de los componentes principales}

Se efectuó un análisis multivariado por medio del estudio de los componentes principales entre adherencia a la alimentación, monitoreo glucémico, actividad física, cuidado de los pies, medicación, consumo de tabaco y características como la antigüedad de la enfermedad, el sexo y el tipo de tratamiento de los encuestados con DM2 (Figura 2). Se utilizaron grupos definidos por sexo y tipo de tratamiento. Es decir que se observó en cada punto del gráfico no un individuo de la muestra, sino un grupo de individuos del mismo sexo y con la misma recomendación de tratamiento. El objetivo fue evaluar un patrón de conducta respecto de la adherencia en función del sexo y el tratamiento de los pacientes. Para la construcción de este conjunto de variables se utilizó la matriz de correlaciones de las variables originales dado que podría ocurrir que la variabilidad de una de las variables fuera mayor a las de las otras y captara representatividad en los componentes principales. Se seleccionaron dos componentes porque entre ambos lograron cubrir el $70,9 \%$ de la variabilidad del conjunto. El primer componente principal logró captar el 19,7\% de la variabilidad del conjunto, mientras que el segundo el $51,2 \%$ de la variabilidad del conjunto.

Entre los dos primeros componentes principales se logró explicar el $70 \%$ de la variabilidad total del conjunto. Se observaron correlacionados en forma positiva: las adherencias a la medicación, al monitoreo, el cuidado de los pies y la alimentación. La antigüedad de la diabetes se asoció de forma positiva con las adherencias mencionadas, sin embargo la edad se relacionó de forma negativa con estas adherencias. Los pacientes con mayor adhesión resultaron pertenecer al grupo de las mujeres bajo tratamiento con antidiabéticos orales e insulina (ambas) y los hombres con insulinoterapia sola, seguido del grupo de mujeres con insulinoterapia solamente y el grupo de hombres con ambas (insulina y antidiabéticos orales). Los menos adheridos pertenecieron al grupo tratados sólo con dieta y ejercicio para ambos sexos. La adherencia a la actividad física resultó opuesta a la adherencia al score de fumador y ambos fueron independientes de las restantes adherencias.

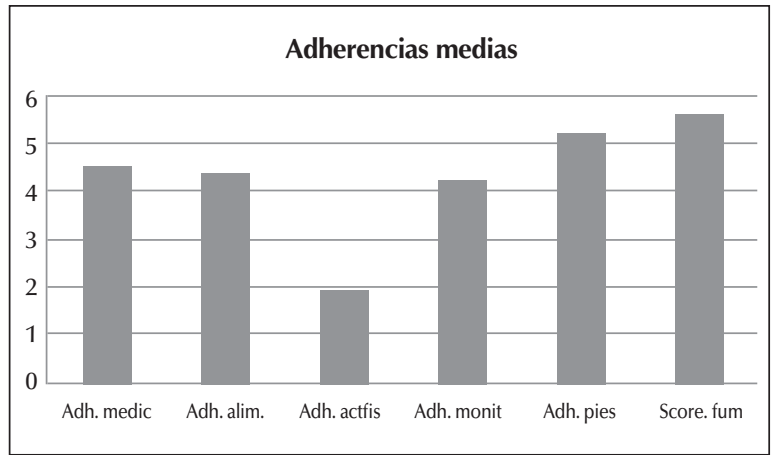

Fuente: elaboración propia.

Adh. medic: adherencia a medicación; adh. alim: adherencia alimentación; adh. act fis: adherencia actividad física; adh. monit: adherencia monitoreo glucémico; adh pies: adherencia al cuidado de los pies; score fumador: adherencia a no fumar.

Figura 1: Escala de adherencias según factores relativos al tratamiento.

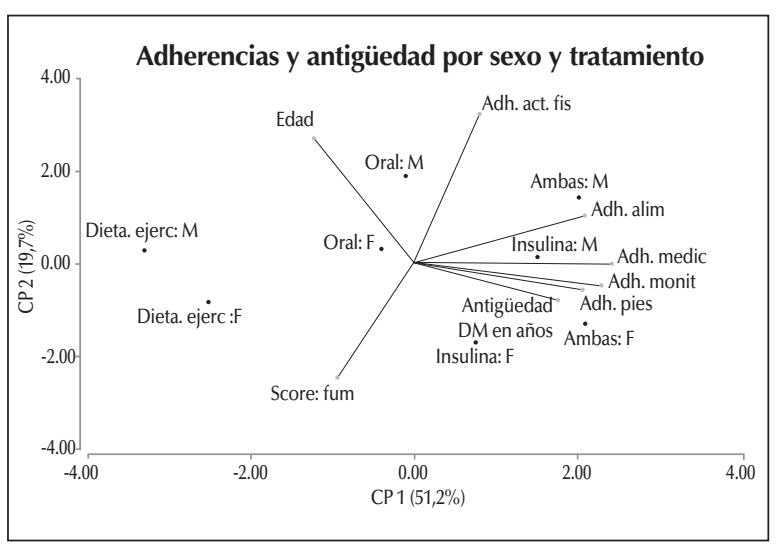

Fuente: elaboración propia.

Adh alim: adherencia alimentaria; adh. act. fis: adherencia a actividad física; adh. monit: adherencia a monitoreo glucémico; adh. pies: adherencia a cuidado de los pies; edad: edad en años; F: sexo femenino; M: sexo masculino; antigüedad DM: antigüedad de la enfermedad; dieta, ejerc: dieta y ejercicio; oral: con antidiabéticos orales; insulina: insulinoterapia; ambas: en tratamiento antidiabético oral e insulina.

Figura 2: Análisis de componentes principales.

\begin{tabular}{|l|c|c|c|}
\hline \multicolumn{1}{|c|}{ Factores de cuidado } & Medias & DS & IC $(95 \%)$ \\
\hline Adherencia medicación & 4,54 & 1.99 & $(4,44 ; 4,64)$ \\
\hline Adherencia alimentación & 4,4 & 2 & $(4,29 ; 4,49)$ \\
\hline Adherencia actividad fisica & 1,94 & 2.18 & $(1,83 ; 2,05)$ \\
\hline $\begin{array}{l}\text { Adherencia monitoreo } \\
\text { glucémico }\end{array}$ & 4,24 & 2.71 & $(4,1,4,37)$ \\
\hline Adherencia cuidado de los pies & 5,22 & 2.06 & $(5,12 ; 5,32)$ \\
\hline Tabaquismo (inversa) & 5,63 & 2.11 & $(5,53 ; 5,74)$ \\
\hline
\end{tabular}

Fuente: elaboración propia.

Media general de adherencia para todo el grupo (M): 4,327(61,71\%,) DS: desvío estándar.

Tabla 1: Resultados en la adherencia según factores relacionados con el cuidado de la DM2. 


\begin{tabular}{|l|l|l|l|l|l|l|l|}
\hline \multicolumn{1}{|c|}{ Adherencia, escala } & \multicolumn{2}{c|}{ OS } & \multicolumn{2}{c|}{ SOS } & \multicolumn{2}{c|}{ Jubilado } & \multicolumn{2}{c|}{ P (diferencias entre grupos de prestación) } \\
\hline Alimentación: M, DS & 4,65, & 1,84 & 4,11, & 2,06 & 4,43, & 2,05 & 0,$0002 ;$ OS y Jub-SOS \\
\hline Actividad física: M, DS & 2,12, & 2,11 & 2,18, & 2,35 & 1,58, & 2,04 & $<<0,0001$ SOS y Jub-OS \\
\hline Monitoreo glucémico: M, DS & 4,7, & 2,45 & 3,83, & 2,97 & 4,19, & 2,63 & 0,$0001 ;$ OS-Jub y SOS \\
\hline Cuidado de pies: M, DS & 5,23, & 1,92 & 5,27, & 2,18 & 5,17, & 2,08 & 0,15 \\
\hline Tabaquismo: M, DS & 5,42, & 2,25 & 5,44, & 2,24 & 5,99, & 1,79 & $<<0,0001$ Jub y OS- SOS \\
\hline Medicación: M, DS & 4,77, & 1,79 & 4,51, & 2,13 & 4,37, & 2 & 0,$01 ;$ OS y Jub \\
\hline
\end{tabular}

Fuente: elaboración propia.

Media general de adherencia para todo el grupo (M): 4,327. OS: obra social; SOS: sin obra social; Jub: jubilado.

Tabla 2: Resultados según escala de adherencia cuantitativa por tipo de prestación.

\begin{tabular}{|c|c|c|c|c|c|c|c|}
\hline Región & Unidad & Medicación & Alimentación & Actividad física & Monitoreo & Cuidado de los pies & TBQ \\
\hline \multirow{5}{*}{$\begin{array}{l}\text { Buenos } \\
\text { Aires }\end{array}$} & Media & 4,47 & 4,21 & 1,94 & 4,04 & 5,28 & 5,76 \\
\hline & DS & 2,04 & 2,04 & 2,31 & 2,88 & 2,12 & 2,08 \\
\hline & Mediana & 4,67 & 4,75 & 1 & 4,5 & 6 & 7 \\
\hline & Q1 & 4,67 & 3 & 0 & 0,88 & 4,2 & 6 \\
\hline & Q3 & 5,42 & 5,56 & 3,5 & 7 & 7 & 7 \\
\hline \multirow{5}{*}{ CABA } & Media & 4,87 & 4,65 & 1,84 & 4,5 & 5,29 & 5,51 \\
\hline & DS & 1,75 & 1,77 & 1,93 & 2,61 & 1,84 & 2,04 \\
\hline & Mediana & 4,67 & 5 & 1,5 & 5 & 5,8 & 6 \\
\hline & Q1 & 4,67 & 3,75 & 0 & 3 & 4,2 & 6 \\
\hline & Q3 & 6,67 & 6 & 3 & 7 & 7 & 7 \\
\hline \multirow{5}{*}{ Comahue } & Media & 4,35 & 4,16 & 2,11 & 4,12 & 4,73 & 5,62 \\
\hline & DS & 2,42 & 2,25 & 2,21 & 2,67 & 2,41 & 2,23 \\
\hline & Mediana & 4,67 & 4,5 & 1,5 & 4,75 & 5,4 & 7 \\
\hline & Q1 & 2,58 & 2,44 & 0 & 2 & 3 & 6 \\
\hline & Q3 & 7 & 6 & 3,5 & 7 & 7 & 7 \\
\hline \multirow{5}{*}{ Centro } & Media & 4,35 & 4,16 & 2,11 & 4,12 & 4,73 & 5,62 \\
\hline & DS & 2,42 & 2,25 & 2,21 & 2,67 & 2,41 & 2,23 \\
\hline & Mediana & 4,67 & 4,5 & 1,5 & 4,75 & 5,4 & 7 \\
\hline & Q1 & 2,58 & 2,44 & 0 & 2 & 3 & 6 \\
\hline & Q3 & 7 & 6 & 3,5 & 7 & 7 & 7 \\
\hline \multirow{5}{*}{ Cuyo } & Media & 4,37 & 4,54 & 2,16 & 4,46 & 5,3 & 5,49 \\
\hline & DS & 2,08 & 2,04 & 2,39 & 2,74 & 2,13 & 2,27 \\
\hline & Mediana & 4,67 & 5 & 1,5 & 5,25 & 6,2 & 6 \\
\hline & Q1 & 4,25 & 3,44 & 0 & 2 & 4,2 & 6 \\
\hline & Q3 & 4,67 & 6 & 3,5 & 7 & 7 & 7 \\
\hline \multirow{5}{*}{ Litoral } & Media & 4,47 & 4,15 & 2,47 & 3,71 & 5,09 & 5,30 \\
\hline & DS & 1,86 & 2,05 & 3,80 & 2,80 & 1,91 & 2,37 \\
\hline & Mediana & 4,67 & 4,50 & 0,00 & 3,75 & 5,60 & 6,00 \\
\hline & Q1 & 4,33 & 2,75 & 0,00 & 0,50 & 4,20 & 6,00 \\
\hline & Q3 & 4,67 & 5,75 & 4,00 & 7,00 & 7,00 & 7,00 \\
\hline \multirow{5}{*}{$\mathrm{NOE}$} & Media & 4,67 & 4,16 & 2,63 & 4,3 & 5,84 & 6,1 \\
\hline & DS & 1,55 & 1,73 & 2,09 & 2,42 & 1,84 & 1,98 \\
\hline & Mediana & 4,67 & 5 & 1,5 & 5 & 5,6 & 6 \\
\hline & Q1 & 4,67 & 3,75 & 0 & 3 & 4 & 6 \\
\hline & Q3 & 5,33 & 6,06 & 3,5 & 7 & 7 & 7 \\
\hline \multirow{5}{*}{ NE } & Media & 4,8 & 4,8 & 1,97 & 4,53 & 5,26 & 5,63 \\
\hline & DS & 1,55 & 1,73 & 2,09 & 2,42 & 1,84 & 1,98 \\
\hline & Mediana & 4,67 & 5 & 1,5 & 5 & 5,6 & 6 \\
\hline & Q1 & 4,67 & 3,75 & 0 & 3 & 4 & 6 \\
\hline & Q3 & 5,33 & 6,06 & 3,5 & 7 & 7 & 7 \\
\hline
\end{tabular}

Fuente: elaboración propia.

DS: desvío estándar; Q1: cuartil inferior; Q3: cuartil superior; TBQ: tabaquismo (inversa); CABA: Ciudad Autónoma de Buenos Aires.

Tabla 3: Escala de adherencia cuantitativa según región determinada geográficamente (correspondiente a los Capítulos de Trabajo de la Sociedad Argentina de Diabetes). 


\begin{tabular}{|l|l|}
\hline Tipo de tratamiento & \multicolumn{1}{|c|}{ Adherencias: $\mathbf{X}, \mathbf{D S}, \mathbf{M}, \mathbf{Q} 1$ y Q3 } \\
\hline \multirow{5}{*}{ Dieta y ejercicio } & Adh. alimentación: 3,$42 ; 2,32 ; 3,5(1,75 ; 5,25)$ \\
& Adh. monitoreo: 2,$98 ; 2,86 ; 3(0 ; 6)$ \\
& Adh. medicación: 2,$63 ; 2,48 ; 2,33(0 ; 4,67)$ \\
& Adh. cuidado pies: 4,$18 ; 2,65 ; 4,6(2 ; 7)$ \\
& Adh. actividad física: 1,$69 ; 2,21 ; 0,5(0 ; 3)$ \\
& Escala fumadores: 5,$82 ; 2,15 ; 7(6 ; 7)$ \\
\hline \multirow{5}{*}{ ADO } & Adh. alimentación: 4,$56 ; 1,91 ; 5(3,5 ; 6)$ \\
& Adh. monitoreo: 3,$65 ; 2,65 ; 3,5 ;(1 ; 6,5)$ \\
& Adh. medicación: 4,18; 1,$38 ; 4,67(4,67 ; 4,67)$ \\
& Adh. cuidado pies: 5,$34 ; 1,93,6(4,2 ; 7)$ \\
& Adh. actividad física: 2,$09 ; 2,25 ; 1,5(0 ; 3,5)$ \\
& Escala fumadores: 5,$64 ; 2,11 ; 6(6 ; 7)$ \\
\hline \multirow{5}{*}{ Insulina y ADO } & Adh. alimentación: 4,$45 ; 1,89 ; 4,75(3,44 ; 6)$ \\
& Adh. monitoreo: 5,$33 ; 2,27 ; 7(4 ; 7)$ \\
& Adh. medicación: 6,$15 ; 1,69 ; 7(6,25 ; 7)$ \\
& Adh. cuidado pies: 5,$47 ; 1,86 ; 6,2(4,4 ; 7)$ \\
& Adh. Actividad física: 2,$02 ; 2,15 ; 1,5(0 ; 3.5)$ \\
& Escala fumadores: 5,$57 ; 2,04 ; 6(6 ; 7)$ \\
\hline \multirow{5}{*}{ Insulina } & Adh. alimentación: 4,$48 ; 2 ; 4,75(3,25 ; 6)$ \\
& Adh. monitoreo: 5,$17 ; 2,49 ; 7(3,5 ; 7)$ \\
& Adh. medicación: 4,$72 ; 1,96 ; 4,67(4,33 ; 7)$ \\
& Adh. cuidado pies: 5,$22 ; 2,05 ; 6(4 ; 7)$ \\
& Adh. actividad física: 1,$65 ; 2 ; 1(0 ; 3)$ \\
& Escala fumadores: 5,$57 ; 2,16 ; 6(6 ; 7)$ \\
\hline
\end{tabular}

Fuente: elaboración propia.

Adh. alimentación: adherencia alimentaria; adh. actividad física: adherencia a actividad física; adh. monitoreo: adherencia a monitoreo glucémico; adh. cuidado pies: adherencia a cuidado de los pies; ADO: antidiabéticos orales.

Tabla 4: Escala de adherencias según tipo de tratamiento prescrito.

\begin{tabular}{|l|l|l|}
\hline \multicolumn{1}{|c|}{ Adherencia } & \multicolumn{1}{|c|}{ Valor $\mathbf{p}$} & $\begin{array}{l}\text { Diferencias entre grupos de } \\
\text { tratamiento prescripto }\end{array}$ \\
\hline Alimentación & $\mathrm{p}<<0,0001$ & $\begin{array}{l}\text { Todos significativamente distintos } \\
\text { entre sí. Escala mayor para } \\
\text { tratamiento oral }\end{array}$ \\
\hline Monitoreo glucémico & $\mathrm{p}<<0,0001$ & $\begin{array}{l}\text { Escala mayor para tratamiento con } \\
\text { insulina e insulina + ADO }\end{array}$ \\
\hline Actividad física & $\mathrm{p}: 0,015$ & $\begin{array}{l}\text { Escalas más bajas para dieta } \\
\text { y ejercicio }\end{array}$ \\
\hline Pies & $<0,0001$ & $\begin{array}{l}\text { El grupo dieta y ejercicio resultó } \\
\text { significativamente distinto de los } \\
\text { restantes y con menor escala }\end{array}$ \\
\hline Medicación & $\mathrm{p}<<0,0001$ & $\begin{array}{l}\text { Todos significativamente } \\
\text { diferentes entre sí. Escala mayor } \\
\text { para ADO e insulina }\end{array}$ \\
\hline Tabaquismo & $\mathrm{p}: 0,0005$ & $\begin{array}{l}\text { El grupo de dieta y ejercicio resultó } \\
\text { significativamente distinto de los } \\
\text { restantes y con mayor escala }\end{array}$ \\
\hline
\end{tabular}

Fuente: elaboración propia.

$P$ : valor de significancia; ADO: antidiabéticos orales.

Tabla 5: Adherencias medias para los distintos tipos tratamientos prescriptos.

\section{DISCUSIÓNY CONCLUSIONES}

En relación con la adherencia encontrada en otras investigaciones sobre estudios con consistencia y pertenencia que aplicaron las encuestas SDSCA, para el estudio de Toobert en el año 2000 la escala media correspondió a 4,31, es decir un $61,9 \%$ de adherencia con valores más bajos para la actividad física, el cuidado de los pies, la alimentación y el monitoreo glucémico ${ }^{12}$. En el estudio realizado en Cartagenas, en 2008, la media total de adherencia fue 4,6 lo que correspondió al $66 \%$ de la adherencia ${ }^{3}$; las escalas más bajas en este estudio se relacionaron con la actividad física, el monitoreo glucémico, la alimentación y el cuidado de los pies. En el trabajo realizado en UOM $2013^{13}$ la media fue de 4,5 , es decir un $64,28 \%$ de adherencia; las escalas más altas correspondieron para la adherencia antitabaco y el cuidado de los pies, y las más bajas para la adherencia a la actividad física, el monitoreo glucémico y la dieta. Debe tenerse en cuenta para este caso que se trataba de pacientes adultos con DM2, con obra social y residentes en el Gran Buenos Aires. La OMS señaló como objetivo de adherencia esperable para países en desarrollo un $70 \%$ de adherencia total, a su vez refirió que un $50 \%$ de los pacientes que padece enfermedades crónicas no cumple con metas objetivo para los valores referidos de adherencia en el tratamiento prescripto'. En este estudio de adherencia el promedio para todos los factores considerados fue de 4,32 $(61,71 \%)$. Los peores puntajes para la adherencia correspondieron a la actividad física, el monitoreo glucémico y la dieta, mientras que los mejores puntajes fueron para la abstención al tabaco, el cuidado de los pies y la toma de medicación. En relación con este objetivo de análisis, en coincidencia con la evidencia, se reconoce que los elementos álgidos en el logro de los niveles óptimos de adherencia lo constituyen la actividad física, el monitoreo glucémico y la dieta.

Un estudio publicado en Diabetes Care en $2011^{16}$ refirió mayor adherencia, percepción personal de control y mejor control glucémico en aquellos que se encontraban bajo tratamiento con insulina. El estudio realizado en la UOM en 2013 detectó que los pacientes (tanto hombres como mujeres) bajo tratamiento con insulina y con insulina, más medicación oral (ambas) en forma conjunta tenían mayor adhesión y menor adhesión en el grupo de tratados sólo con dieta y ejercicio para ambos sexos, resultados coincidentes con el es- 
tudio multicéntrico ${ }^{12}$. Se detectaron resultados tal vez redundantes como la mayor adherencia al monitoreo en las terapias de uso con insulina y menores para dieta y ejercicio y sólo uso de ADO, como así también la mayor adherencia en la medicación de terapias con insulina e insulina más ADO. Son alarmantes las bajas escalas referidas al grupo con sólo dieta y ejercicio.

En relación con las limitaciones, respecto de la población objetivo y la muestra alcanzada, hubo una región que superó el $20 \%$ de rechazo según el objetivo propuesto que correspondió a la región Centro (Córdoba con un rechazo del 65,64\%). El resto de las regiones que no cubrió el número objetivo de 165 no alcanzó el 20\% del rechazo (Anexo). A pesar de lo mencionado, para la muestra en total el faltante representó sólo un 2,5\%. Respecto de los datos relacionados a los antecedentes de comorbilidades y complicaciones de la enfermedad, se analizó según lo referido por los pacientes y esto podría subestimar los resultados observados. El tipo de encuesta transversal reflejó sólo lo referido para ese tiempo encuestado por lo que pueden encontrarse diferencias estadísticamente significativas en el cumplimiento de los objetivos de control metabólicos en tiempos anteriores para algunos de esos pacientes (la encuesta hacía referencia solo a la HbA1c y los promedios de glucemia desde el momento de realizada la misma tres meses hacia atrás en el tiempo). En el estudio no se contempló la variable educación diabetológica recibida y por consiguiente no pudo más que analizarse el nivel de educación. No debe subestimarse un posible error aleatorio asociado a una muestra de tamaño limitado dado que no se contemplaron conglomerados de región detalladamente representativos.

La falta de adherencia del paciente es una amenaza constante para la salud y la calidad de vida. Los resultados de adherencia encontrados se asemejan a otros países en desarrollo a excepción del cuidado de los pies. El factor cuidado de los pies en nuestro país, en los estudios hasta la fecha referidos, reportan escalas de mayor adherencia. No obstante individualizar por antecedentes personales denota diferencias vinculadas al acceso, el sexo, la edad y el nivel de educación.

Existen elementos o herramientas que exceden lo económico y son fundamentales para realizar un cambio de comportamiento en los pacientes con DM2. Se necesitan políticas públicas y privadas para reducir la brecha de accesibilidad y promover hábitos saludables que mejoren especialmente aquellas variables de cuidado de tratamiento que cuantifican más bajo a nivel mundial como la actividad física, el monitoreo glucémico y la alimentación. Considerar intervenir precoz y especialmente en el grupo de pacientes bajo tratamiento con alimentación y actividad física solamente (grupo que cuantificó más bajo en la mayoría de los factores de cuidado de la DM2 salvo para la actividad física) es fundamental para prevenir complicaciones y el agregado de comorbilidades futuras mediatas.

El estudio demostró una considerable consistencia. En relación con la aplicación del cuestionario SDSCA, es una medida multidimensional factible para evaluar autogestión y viable de extrapolarse en diferentes subpoblaciones con diabetes. La distribución de las mediciones debe basarse en la estabilidad de los comportamientos de adhesión y la congruencia temporal con otras medidas de interés como, por ejemplo, los objetivos de control glucémico.

Argentina se halla en similar rango de cumplimiento en relación con estudios previos $(61,84 \%)^{13}$. Individualizar cualitativamente los motivos de incumplimiento permite conocer sus causas principales como la falta de educación y los problemas económicos. Intervenir específicamente sobre estas causas podría reducir el impacto negativo de la falta de adherencia al tratamiento. Con esta investigación, al evaluar la adherencia en forma multicéntrica, se lograron conocer los factores significativos relativos al cuidado de la DM2 y su tratamiento. Se aplicó un método validado de evaluación indirecto, confiable y viable de aplicar, adaptado culturalmente que logra plasmar parte de la situación actual y el estado del problema en forma real. El estudio multicéntrico realizado, si bien seleccionó la muestra para todos los pacientes que desearan y pudieran participar sin contemplar conglomerados de región del todo representativos comparados con otras características de la demografía de regiones, logró una correlación de la situación actual entre la adherencia y las variables individuales en la totalidad y por regiones.

\section{BIBLIOGRAFÍA}

1. OMS, 2004. Adherencia a los tratamientos a largo plazo: pruebas para la acción. Ginebra (Internet) 2004. Citado en marzo 2015. Disponible en: http://www.paho.org/Spanish/AD/DPC/ $\mathrm{NC} / \mathrm{nc}$-adherencia.html a largo plazo. 
2. Información farmacoterapéutica de la Comarca (INFAC). Adherencia al tratamiento farmacológico en patologías crónicas. Liburukia 2011; 9(1) (Internet) 2014. Citado en agosto 2017. Disponible en: https://www.osakidetza.euskadi.eus/contenidos/informacion/cevime_infac/es_cevime/adjuntos/INFAC_v19_n3.pdf

3. Alayón A, Mosquera-Vásquez M. Adherencia al tratamiento basado en comportamientos en pacientes diabéticos. Cartagena de Indias, Colombia. Rev Salud Pública 2008; 10(5):777-787.

4. Morisky DE. Adherence or compliance behavior, encyclopedia of public health. 2010 (Internet 2017). Citado en 2017. Disponible en: URL: http://www.encyclopedia.com/doc/1G2-3404000026.html.

5. Haynes RB, Ackloo E, Sahota N, et al. Interventions for enhancing medication adherence. Cochrane Database of Syst Rev 2008; 16 ; (2):CD000011. Doi: 10.1002/14651858.CD000011.

6. NICE. National Institute for Health and Clinical Excellence. Medicine adherence: involving patient indecisions about prescribed medicines and supporting adherence. Clinical Guideline 2009. (Internet 2017). Citado en agosto 2017. Disponible en: https://www.scribd.com/document/200320735/NICE-Guidelines-Regarding-Adhrence.

7. Arribas M. Diseño y validación de cuestionarios. Matronas Profesión 2004; 5(1): 23-29.

8. Mendioca E. Manual teórico práctico de investigación social. Buenos Aires. Espacio. 2004; 5: 67-74.

9. Mendioca GE. Sobre tesis y tesistas. Buenos Aires. Espacio. 2003; 6: 106-124.

10. Morisky DE, Green LW, Levine DM. Concurrent and predictive validity of a self-reported measurements of medication adherence and long term predictive validity of blood pressure control. Med Care 1986; 24(1): 67-74.
11. Morisky DE, Ang A, Krousel Wood M, et al. Predictive validity of a medication adherence measure in an outpatient setting. $J$ Clin Hypertension (Greenwich) 2008; 5:348-354

12. Toobert D, Hampson S, Glasgow R. The summary of diabetes self-care activities measure: Results from 7 studies and a revised scale. Diabetes Care 2000; 23(7): 943-950.

13. Linari MA. Factores que se asocian a la adherencia al tratamiento en diabetes mellitus tipo 2 en adultos del Gran Buenos Aires pertenecientes a la Unión Obrera Metalúrgica, seccional Vicente López, durante 2013. Tesis de Maestría en Epidemiologia, Gestión y Políticas de Salud. Universidad Nacional de Lanús, 2015. Recuperado a partir de: http:www.unla.edu.ar/index. php/servicios-de-la-biblioteca.

14. Linari MA, González C, Alvariñas J. Desarrollo y validación de un cuestionario sobre adherencia al tratamiento y costo de bolsillo en pacientes adultos con diabetes mellitus tipo 2. Revista de la Sociedad Argentina de Diabetes. Trabajos presentados en el XVII Congreso Argentino de Diabetes 2010; 44(4): 336-337.

15. Inzucchi SE, Bergenstal RM, Buse JB, Diamant M, Ferrannini E, Nauck M, Peters AL, Tsapas A, Wender R, Matthews DR; American Diabetes Association (ADA); European Association for the Study of Diabetes (EASD). Management of hyperglycemia in type 2 diabetes: a patient centered approach. Position statement of the American Diabetes Association (ADA) and the European Association for the Study of Diabetes (EASD) Diabetes Care 2012; 35(6):1364-79.

16. Broadbent E, Donkin P, Stroh JC, Psych D. Illness and treatment perceptions are associated with adherence to medications, diet, and exercise in diabetic patients. Diabetes Care 2011; 34 (2):338-340.

\section{ANEXO}

\begin{tabular}{|l|l|l|}
\hline \multicolumn{1}{|c|}{ Región } & \multicolumn{1}{c|}{ N } & \% \\
\hline Buenos Aires & 261 & 17,17 \\
\hline CABA & 167 & 10,99 \\
\hline Comahue & 224 & 14,74 \\
\hline Centro & 67 & 4,41 \\
\hline Cuyo & 229 & 15,07 \\
\hline Litoral & 188 & 12,37 \\
\hline Noroeste & 140 & 9,21 \\
\hline Noreste & 244 & 16,05 \\
\hline
\end{tabular}

\begin{tabular}{|l|l|l|}
\hline \multicolumn{1}{|c|}{ Cobertura } & \multicolumn{1}{|c|}{ N } & \% \\
\hline Jubilado & 554 & 36,45 \\
\hline OS & 481 & 31,64 \\
\hline SOS & 485 & 31,91 \\
\hline
\end{tabular}

Fuente: elaboración propia

OS: con obra social; SOS: sin obra social;

N: número de encuestados.

Tabla 2: Distribución de encuestados según cobertura en salud.

Fuente: elaboración propia.

N: número de encuestados; CABA: Ciudad Autónoma de

Buenos Aires.

Tabla 1: Descripción de la muestra según región (1.520 reclutados). 


\begin{tabular}{|c|c|c|c|}
\hline Variable & OS & SOS & Jubilado \\
\hline Sexo F/M & $240 / 234$ & $256 / 218$ & $306 / 233$ \\
\hline Edad: M, DS & $56,59(12,04)$ & $54,38(11,64)$ & $69,87(9,53)$ \\
\hline Antigüedad DM2: (años) & $8,95(6,82)$ & $8,85(7,6)$ & $12,64(10,5)$ \\
\hline \multicolumn{4}{|l|}{ Estudios: (N, \%) } \\
\hline $\mathrm{NL}$ & $3(0,62)$ & $13(2,68)$ & $21(3,79)$ \\
\hline $\mathrm{Pl}$ & $35(7,27)$ & $111(22,88)$ & $123(22,20)$ \\
\hline PC & $99(20,58)$ & $123(25,36)$ & $189(34,11)$ \\
\hline SI & $43(8,93)$ & $96(19,79)$ & $66(11,91)$ \\
\hline SC & $112(23,28)$ & $82(16,9)$ & $79(14,25)$ \\
\hline TI-UI & $40(8,31)$ & $23(4,74)$ & $13(2,34)$ \\
\hline TC-UC & $121(25,15)$ & $16(3,29)$ & $32(5,77)$ \\
\hline $\mathrm{NC}$ & $28(5,82)$ & $21(4,32)$ & $31(3,59)$ \\
\hline \multicolumn{4}{|l|}{ Antecedentes N, \% } \\
\hline HTA & $223 / 257(53,54 \%)$ & $187 / 297(61,36 \%)$ & $181 / 373(67,32 \%)$ \\
\hline EC & $426 / 55(11,43)$ & $420 / 65(13,30)$ & 460/94 $(16,96)$ \\
\hline DLP & $255 / 226(46,98)$ & $237 / 248(51,13)$ & $282 / 272(49,09)$ \\
\hline RTN & $371 / 110(22,86)$ & $285 / 200(41,23)$ & $370 / 184(33,21)$ \\
\hline IR & $461 / 20(4,15)$ & $416 / 67(13,87)$ & $490 / 64(11,55)$ \\
\hline Neuropatia & $416 / 65(13,51)$ & $341 / 144(42,22)$ & $433 / 121(21,84)$ \\
\hline Alt. hepáticas & $445 / 36(7,84)$ & $429 / 56(13,05)$ & $482 / 72(14,93)$ \\
\hline $\mathrm{HbA1c}$ & $7,45(1,56)$ & $8,22(4,36)$ & $7.88(9,37)$ \\
\hline Glucemias mg/dl: M, DS & $140,88(49,8)$ & $164,98(63,49)$ & $145,17(42,91)$ \\
\hline $\begin{array}{l}\text { Objetivos de control } \\
\text { cumpl /no cumpl: N, \% }\end{array}$ & $260 / 150(63,41)$ & $197 / 196(50,12)$ & $145,17(42,91)$ \\
\hline
\end{tabular}

Fuente: elaboración propia.

OS: obra social; SOS: sin obra social; F: femenino; M: masculino; DS: desvío estándar; NL: no lee ni escribe; Pl: primario incompleto; PC: primario completo; SI: secundario incompleto; SC: secundario completo; TI: terciario incompleto; TC: terciario completo; UI: universitario incompleto; UC: universitario completo; NC: no contestó; HTA: hipertensión arterial; EC: eventos cardiovasculares; DLP: dislipemia; RTN: complicaciones oculares; IR: complicaciones renales; alt. hepat.: trastornos hepáticos; cumpl: cumplió con objetivos.

Tabla 3: Antecedentes demográficos y características clínicas. 